\title{
INTEGRACIÓN PEDAGÓGICA DE LA TECNOLOGÍA INFORMÁTICA EN INSTITUCIONES EDUCATIVAS OFICIALES DE CARTAGENA DE INDIAS (COLOMBIA)*
}

\author{
PEDAGOGICAL INTEGRATION OF COMPUTER TECHNOLOGY IN \\ PUBLIC SCHOOLS OF CARTAGENA DE INDIAS (COLOMBIA)
}

\author{
Miguel Garcés Prettel ${ }^{1}$ \\ Rosmayra Ruiz Cantillo ${ }^{2}$
}

\begin{abstract}
RESUMEN
Se identificaron las dinámicas de integración y uso de la tecnología informática en las labores de docencia de instituciones educativas oficiales de Cartagena de Indias (Colombia) que han sido beneficiarias del programa presidencial "Computadores para Educar". La investigación fue de tipo descriptivo, con una muestra de 350 docentes de 27 escuelas del distrito. El instrumento utilizado es una adaptación del cuestionario de Jaramillo, Castañeda y Pimienta (2009), complementado con categorías sobre logros y dificultades en la inserción de estas tecnologías dentro de los procesos académicos. Los hallazgos revelan múltiples tipologías de docencia (personalista, sistematizador, documentador, expositor, participativo y pragmatista) a partir de los modos de integrar estos recursos en el aula, como también se revelan las diversas estrategias de uso, algunas de las cuales eran percibidas como generadoras de cambios pedagógicos, mientras otras eran consideradas viejas metodologías con nuevas tecnologías al servicio de la educación tradicional.
\end{abstract}

\section{PALABRAS CLAVE}

tecnología de la información, docencia, estrategia de enseñanza.

\begin{abstract}
The dynamics of integration and use of information technology were identified in the teaching work of official educational institutions in Cartagena de Indias (Colombia), which have been beneficiaries of the presidential program "Computadores para Educar." The research was descriptive, with a sample of 350 teachers from 27 schools in the district. The instrument used is an adaptation of the questionnaire of Jaramillo, Castañeda and Pimienta (2009), complemented with categories on achievements and difficulties in the insertion of these technologies within the academic processes. The findings reveal multiple types of teaching (personal, systematizing, documentary, exhibitor, participatory and pragmatist) from the ways of integrating these resources in the classroom, as well as the various strategies of use, some of which were perceived as generators of pedagogical changes, while others were considered old methodologies with new technologies in the service of traditional education
\end{abstract}

\section{KEYWORDS}

Information technology, teaching profession, teaching strategy.

Fecha de recepción: 2 de febrero de 2016.

Fecha de evaluación: 9 de marzo de 2016.

Fecha de aceptación: 19 de abril de 2016.

* Esta investigación fue financiada dentro del maro de la convocatoria 525 de 2011 liderada por el Departamento Administrativo de Ciencia y Tecnología - Colciencias.

1 Magister en Comunicación. Profesor asociado Universidad Tecnológica de Bolívar. Investigador del Grupo de Estudios Sociales Humanísticas de la Universidad Tecnológica de Bolívar. Miembro de la Red Iberoamericana de Pedagogía. Correo electrónico: mgarces@ unitecnologica.edu.co. Dirección postal: Km 1 Vía Turbaco-Bolívar (Colombia). Tel. (57)-5-6535200 ext. 262

2 Comunicadora Social, Universidad Tecnológica de Bolívar. megp_mg@hotmail.com. Dirección postal: Universidad Tecnológica, Km 1 Vía Turbaco-Bolívar (Colombia). Tel. (57)-5-6535200 ext. 262 


\section{INTRODUCCIÓN}

En marzo de 2007 se realizó en Buenos Aires (Argentina) la segunda reunión intergubernamental de Ministros de Educación del Proyecto Regional de Educación para América Latina y el Caribe auspiciado por la Organización de las Naciones Unidas para la Educación, la Ciencia y la Cultura (UNESCO). El tema central fue la educación de calidad como derecho humano fundamental, con el fin de reducir o eliminar la exclusión social asegurando la igualdad de oportunidades en el acceso al conocimiento.

Una de las recomendaciones a nivel político enfatizó la relación entre exclusión social y brecha tecnológica, sugiriendo la necesidad de desarrollar escuelas con aulas diversificadas, implementar sistemas de apoyo para atender las dificultades de aprendizaje y proveer recursos informáticos a los estudiantes para mejorar los aprendizajes y la equidad (UNESCO, 2007).

Colombia atendió este desafío no solo con la puesta en marcha del plan nacional de tecnologías de la información y la comunicación, sino que también fortaleció el programa presidencial "Computadores para Educar" (CPE) que venía desde el año 2000 trabajando con instituciones educativas oficiales de las distintas regiones de Colombia con el fin de superar el analfabetismo digital.

Desde su génesis, el programa busca reducir las brechas sociales recolectando equipos de cómputo dados de baja por empresas públicas y privadas de Colombia y del exterior, para su reacondicionamiento y posterior entrega sin costos a las instituciones educativas oficiales de Colombia (Mejía y Bernal, 2003).

Se han hecho estudios para sistematizar el impacto que ha tenido este programa, entre los que sobresalen: los diagnósticos que arrojan un alto índice de satisfacción de los beneficiarios con el programa (Computadores para Educar, 2012); las mediciones que han demostrado que incrementa el uso de estos recursos informáticos en las instituciones educativas beneficiarias (Barrera y Linden, 2009); además, existe evidencia de que el programa en determinadas condicio- nes disminuye la tasa de deserción, mejora los puntajes de las pruebas de Estado y aumenta la probabilidad de ingreso a la educación superior (Rodríguez, Sánchez y Márquez, 2011).

Sin embargo, aunque estas investigaciones aportan una mirada general de lo que ha hecho el gobierno colombiano a través de CPE, existe la necesidad social de llenar un vacío de conocimiento sobre lo que ocurre con el aprovechamiento pedagógico de estas tecnologías de la información y la comunicación (TIC) en las instituciones educativas de Cartagena de Indias beneficiarias de este programa, y que son objeto de estudio de esta investigación.

Cartagena de Indias, es una ciudad costera de la región norte del Caribe colombiano, conocida mundialmente por el turismo y por ser declarada en 1984 por la UNESCO como patrimonio histórico y cultural de la humanidad; además es un territorio rodeado de enormes contrastes en lo social, pues a pesar de ser un distrito que alcanza grandes dividendos en materia portuaria, turística, comercial e industrial, las cifras desde el año 2009 revelan "que de cada 100 Cartageneros 36 se encuentran padeciendo la pobreza, y de estos 9 se encuentran en situación de miseria" (PNUD, 2011:30).

Los docentes de las instituciones educativas participantes en este estudio, trabajan precisamente con estudiantes que vienen de estas familias cartageneras vulnerables, muchos de ellos presentan limitaciones económicas no solo para subsistir, sino también para adquirir o acceder a tecnología informática desde sus hogares, generándose una doble exclusión: la exclusión socioeconómica y la exclusión tecnológica, ambas no permiten apropiarse del entorno global que habitan.

Por esas mismas razones, se consideró pertinente realizar esta investigación porque aporta a esos fines sociales de inclusión social y tecnológica que viene liderando el programa CPE, brindando información pertinente a las instituciones educativas cartageneras sobre los usos tecnológicos, incluyendo los logros obtenidos, las limitaciones encontradas y algunas recomendaciones que ayudarían a estas institucio- 
nes al fortalecimiento de sus procesos y políticas internas tendientes a lograr que los docentes hagan un uso eficiente de las TIC y motiven a sus estudiantes a usarlas educativamente.

En ese sentido el objetivo de este estudio partió de dos preguntas relevantes: ¿Cómo integran los docentes las TIC en los procesos pedagógicos que desarrollan en las instituciones educativas de Cartagena de Indias beneficiarias del programa CPE? ¿Qué dificultades y logros pedagógicos se han dado en los procesos de integración de estas tecnologías en las labores de enseñanza-aprendizaje?

Al responderlas, se pudo también determinar las diversas tipologías de docencia mediadas por estas tecnologías

\section{INTEGRACIÓN DE LAS TIC EN LOS PROCESOS EDUCATIVOS}

Si bien el concepto de TIC abarca todos los recursos tecnológicos que facilitan la comunicación y flujo de la información, para el caso de esta investigación el término hace referencia a la tecnología informática suministrada por el programa presidencial "Computadores para Educar", es decir los equipos de cómputo con software y acceso a redes locales e internet que recibieron en donación las instituciones educativas que forman parte de la muestra.

La integración de las TIC en los sistemas educativos, ha sido y sigue siendo, un tema de interés de gobiernos y organizaciones. En América Latina por ejemplo, Artopoulos y Kozak (2011) realizaron una investigación documental sobre las características comunes de las experiencias y proyectos relacionados con el uso de las TIC en el contexto educativo: escenarios centrados en la convivencia de culturas y códigos de comunicación; preocupación por aprender a reconocer y trabajar con los saberes y competencias que traen los estudiantes; se han desarrollado estrategias didácticas basadas en TIC que posibilitan una apropiación significativa de los contenidos curriculares; hay interés en la formulación e implementación de políticas de equipamiento y conectividad; además de un interés en la actualización profesional de los docentes; se han generado espacios físicos; infraestructura, modelos didácticos y prácticas mediadas por TIC en el aula en pro de la inclusión tecnológica.

En este contexto de experiencias, han aparecido múltiples formas de integrar las TIC en los procesos educativos, tal vez las más comunes (que pueden darse gradual o alternadamente) son: la integración para la gestión administrativa, la integración para la gestión pedagógica o curricular, la integración para el diseño e implementación de proyectos pedagógicos.

\subsection{INTEGRACIÓN DE LAS TIC EN LA GESTIÓN ADMINISTRATIVA}

Investigaciones como las de Trahtemberg (2001) y el informe sobre "Implantación y Uso de las TIC en Escuelas de Educación Primaria y Secundaria de España" realizado por el Instituto de Evaluación y Asesoramiento Educativo entre 2005 y 2006 , mostraron que el uso más frecuente de las TIC en la organización escolar está relacionado con la digitalización de procesos de matrícula, la sistematización de expedientes, labores de comunicación con familias, profesores y otros centros educativos.

La integración de las TIC en la gestión administrativa escolar, aunque tiene que ver con los procedimientos administrativos abarca mucho más, al parecer lo esencial en términos de desarrollo educativo estriba en la implementación de un modelo de gestión que sea capaz de interrelacionar lo administrativo, lo logístico y lo financiero al servicio del fortalecimiento curricular o académico, generando políticas internas para lograr usos integrados y eficientes de las TIC en todas las áreas de la institución.

\subsection{INTEGRACIÓN DE LAS TIC EN LA GESTIÓN PEDAGÓGICA O CURRICULAR}

Frente a este aspecto, Sánchez (2003) enmarca este tipo de usos con el propósito de apoyar las disciplinas o contenidos curriculares haciendo del aprendizaje, los objetivos, y las estrategias de individualización y planeación educativa el centro del proceso, y no los recursos tecnológicos. Este autor, definió un método de integra- 
ción a partir del modelo de Fogarty, en donde se reconocen seis maneras de usar las TIC dentro del ámbito curricular:

Forma anidada: cuando en los contenidos de una asignatura los docentes logran que los estudiantes usen las TIC para desarrollar y ejercitar habilidades sociales y de pensamiento, además de aplicar aspectos puntuales del contenido tratado.

Forma tejida: los estudiantes usan las TIC para examinar conceptos e ideas en temas relevantes de una asignatura que están interrelacionados con otras disciplinas y contenidos.

Forma enroscada: aquí se usan las TIC para integrar y fortalecer habilidades sociales, habilidades de pensamiento y de inteligencias múltiples a través de varias disciplinas.

Forma integrada: las TIC son usadas como plataforma de apoyo para unir asignaturas en la búsqueda de superposiciones de conceptos e ideas.

Inmerso: el estudiante filtra el contenido con apoyo de TIC y se apropia de la experiencia profundizando en sus procesos educativos.

En red: el estudiante filtra su aprendizaje con el uso de TIC, generando conexiones internas que lo llevan a interacciones externas con expertos de áreas afines.

En el mismo orden de ideas, Orjuela (2010) realiza una compilación importante de modelos de integración de TIC dentro del ámbito pedagógico que se han usado en diferentes contextos educativos, entre estos resalta:

El nivel de pre-integración: el docente hace uso de las TIC para incrementar su productividad personal en labores comunicación, talleres y exámenes; almacena y organiza información de estudiantes; mantiene registro de calificaciones; se comunica por correo electrónico, y utiliza internet para localizar diversos recursos de las clases.

Instrucción dirigida: el docente utiliza las TIC como herramienta de instrucción programada para que sus estudiantes se entrenen con prácticas, tutoriales y software de ejercicio.

Integración básica: el docente emplea las TIC en la elaboración de materiales para sus estudiantes; además utiliza computadores, software y videobeam para enriquecer sus clases.

Integración media: el docente solicita a los estudiantes utilizar diferentes herramientas informáticas y no informáticas para realizar trabajos de clase.

Integración avanzada: el docente aprovecha las TIC y usa metodologías de aprendizaje activo para realizar proyectos de clase enfocados en el currículo y lograr mejoras en el aprendizaje de sus estudiantes

Así mismo Hooper \& Rieber (1995) señalan las distintas formas o etapas en que los docentes usan las TIC en sus labores o intereses: a) Familiarización: los docentes aprenden a usar el computador y algunos programas, pero solo los utiliza en labores personales; b)Utilización: los docentes usan las TIC en labores educativas de índole administrativa sin articularlas a propósitos pedagógicos; c)Integración: usan las TIC para apoyar su labor docente en tareas y responsabilidades definidas dentro de los planes de curso; d)Reorientación: el docente por medio de las TIC facilita ambientes de aprendizaje para que sus estudiantes construyan saberes o conocimientos; e)Evolución: los docentes actualizan permanente su práctica pedagógica con las nuevas herramientas tecnológicas que van surgiendo y teniendo claro cómo aprenden las personas.

En los procesos de integración de ésta índole, intervienen también los aspectos académicos e institucionales inherentes a la escuela, lo cual quiere decir, que para obtener una mejor evaluación del alcance de las dinámicas de inserción de las TIC en lo curricular y pedagógico, se requieren metodologías que exploren cada uno de esos factores de forma interrelacionada. Batista (2007) considera que las TIC deben usarse de forma transversal sirviendo al logro de objetivos educativos que promuevan la aplicación del conocimiento, la interacción y 
aprendizaje autónomo, además pueden ser usadas estratégicamente para fortalecer el trabajo colaborativo que es una competencia esencial para el aprendizaje (García, Basilotta y López, 2014).

\subsection{INTEGRACIÓN DE LAS TIC EN LA GES- TIÓN DE PROYECTOS PEDAGÓGICOS}

Existen experiencias que demuestran cómo estas tecnologías fortalecen mejor el accionar de los proyectos pedagógicos que tienen como objetivo: aplicar conocimientos para la solución de problemas de aprendizaje, reforzar saberes, o bien para que la comunidad educativa asuma un compromiso transformador con las necesidades del contexto social, cultural, económico, político o ambiental donde están inmersos los centros educativos.

Uno de los trabajos destacables de sistematización de experiencias de proyectos pedagógicos basados en TIC, fue el realizado en Colombia por el Ministerio de Educación Nacional (MEN) con poblaciones vulnerables. El MEN logró atraer 190 experiencias significativas que demostraron como las TIC bajo a esta modalidad, están contribuyendo significativamente a la promoción de la educación intercultural, la etno-identidad, el compromiso socioambiental, el desarrollo artístico, la gestión empresarial y la intervención para mejorar problemas de autismo y déficit cognitivo (Pardo, 2005).

Estas experiencias son formas innovadoras de utilizar los recursos tecnológicos que dispone la escuela para relacionarlo con otros asuntos más allá de la clase tradicional, con el fin de resolver problemas del contexto social y escolar; a su vez, ayuda a fortalecer la cultura escolar mediante la afirmación de los procesos identitarios y el desarrollo de proyectos locales de emprendimiento.

Otro trabajo destacable en este mismo enfoque, es el realizado por Moreno et. al (2011) que mencionan un sinnúmero de experiencias sobre proyectos pedagógicos mediados por TIC, y proponen una metodología especial para sistematizar rigurosamente estos procesos, partiendo de categorías como: el contexto socio- cultural de las comunidades, los enfoques de enseñanza de las áreas de educación básica y media, y las posibilidades educativas que brindan las TIC.

\section{METODOLOGÍA}

Esta investigación de carácter descriptivo-exploratorio se realizó con 27 centros educativos públicos de Cartagena de Indias que recibieron donación tecnológica del programa presidencial "Computadores para Educar". El universo poblacional lo conforman los docentes de las instituciones seleccionadas, tomando como muestra los 350 docentes que laboran en todas las jornadas $(Z=1.96 ; 0.25 ; E=5 \%)$. En la selección muestral se incluyeron docentes de instituciones educativas oficiales beneficiarias de este programa gubernamental en un periodo no menor a dos años. Los docentes participantes tenían al menos un año de estar vinculado a estas escuelas.

Para la recolección de la información se aplicó un cuestionario tipo encuesta dirigido a los docentes, en donde se recogió información sobre los usos personales y educativos de la tecnología informática. La encuesta es una adaptación del instrumento diseñado por Jaramillo, Castañeda y Pimienta (2009) que mide los usos tecnológicos de los docentes. Dicho instrumento fue complementado con nuevos ítems sobre logros y dificultades que percibieron los docentes con la inserción de estas tecnologías en el aula. La encuesta recoge información básica del encuestado, tiene preguntas dicotómicas sobre diversas maneras de usar la tecnología informática; además contiene preguntas abiertas sobre el fomento del uso de estas herramientas entre los estudiantes. Las preguntas cerradas fueron diseñadas a partir de los resultados obtenidos de las entrevistas a directivos y dos grupos focales con docentes quiénes hablaron sobre el alcance educativo logrado con estas tecnologías, los conocimientos que tienen sobre tecnología informática, y los usos pedagógicos de estas tecnologías.

La información fue tabulada con el Software Estadístico SPSS V.18. Los datos fueron categorizados y contados de forma descriptiva, por me- 
dio de los reportes de frecuencia que suministraba el programa a partir de los ítems relacionados con los usos docentes de estas tecnologías y los logros y dificultades pedagógicas percibidas por los docentes en las dinámicas de integración de la tecnología informática. Los cruces de variables hicieron posible caracterizar las tipologías de docencia mediadas por TIC y las estrategias de uso tecnológico más frecuentes.

\section{RESULTADOS}

\subsection{INFORMACIÓN DEMOGRÁFICA Y PROFESIONAL}

El 58\% de los docentes encuestados son de sexo masculino y el $42 \%$ femenino. En cuanto a lo etario se aprecia una buena convergencia generacional, en donde el $5 \%$ está en la etapa de juventud plena ( 20 a 25 años), el resto es población adulta caracterizada así: el $24 \%$ está en la categoría de adultez temprana (26 a 37 años); el $42 \%$ corresponde a adultos intermedios ( 38 a 48 años); el 17\% está en la adultez plena (49 a 54 años) y el 12\% restante está en el rango de adulto mayor.

En los dos grupos había presencia de docentes de las diferentes áreas: Ciencias Sociales, Lengua Castellana, Ciencias Naturales, Matemáticas, Lenguas Extranjeras y Educación Física. En lo concerniente al desempeño docente en la escuela: el 17\% manifestó tener de 1 a 4 años ejerciendo su profesión desde su área de experticia; el 35\% tiene entre 5 a 9 años; el 17\% entre 10 a 14 años de experiencia; el 24\% entre 15 a 20 años y el 6\% tiene más de 20 años. Ambos resultados coinciden con los criterios de selección muestral preestablecido.

\subsection{CONOCIMIENTO TECNOLÓGICO}

La alfabetización tecnológica en la población docente de estas escuelas ha ido fortaleciéndose desde la llegada de las TIC donadas por el programa "Computadores para Educar". En la actualidad el $78 \%$ de los docentes saben usar los computadores y las herramientas informáticas disponibles en estos centros educativos. El origen de estos conocimientos tienen diversas fuentes: personal e institucional.
En lo personal, algunos aprendieron a usar la tecnología informática de forma autodidacta (37\%), otros hicieron posgrado, especializándose en el uso de estos recursos (16\%). A nivel institucional, hay que resaltar los esfuerzos de capacitación organizados por la institucionalidad educativa (47\%) en cabeza del liderazgo del gobierno local, la Secretaría de Educación y del programa "Computadores para Educar", todos ellos han contribuido a que estos docentes se hayan formado en el uso de la tecnología informática. Los docentes de estos centros educativos dentro de sus procesos de alfabetización tecnológica adquirieron una variedad de saberes sobre el uso de programas informáticos que pueden clasificarse así:

Conocimientos en ofimática: nivel básico (29\%), nivel medio (22\%), nivel avanzado $(18 \%)$.

Conocimientos en programa de diseño gráfico: nivel básico (29\%), nivel medio (19\%), nivel avanzado (24\%).

Conocimientos en redes de datos e Internet: nivel básico $(29 \%)$, nivel medio (22\%), nivel avanzado (18\%).

Conocimientos en redes sociales: nivel básico $(22 \%)$, nivel medio (21\%), nivel avanzado (14\%).

Conocimientos en plataformas educativas virtuales: nivel básico (60\%), nivel medio (16\%), nivel avanzado (13\%).

Conocimientos en software educativo: nivel básico $(21 \%)$, nivel medio (16\%), nivel avanzado $(13 \%)$.

Conocimientos en programas de desarrollo: nivel básico (13\%), nivel medio (9\%), nivel avanzado (8\%).

Conocimientos en programas de simulación: nivel básico (17\%), nivel medio (17\%), nivel avanzado ( $8 \%)$.

A pesar de estos avances en el aprendizaje informático, todavía hay que seguir trabajando en 
la tarea de erradicar el analfabetismo informático que persiste en un $22 \%$ de los docentes que aseguran no saber usar computadores, ni ningún otro recurso informático existente en las escuelas. Al relacionar este tipo de analfabetismo con las variables demográficas no se apreció ninguna tendencia hacia un género específico, pues las proporciones de analfabetismo fueron similares tanto en los docentes hombres como en las mujeres.

Caso contrario ocurrió cuando se relacionó descriptivamente con la variable edad, allí como era de esperarse el analfabetismo informático fue mayor en docentes mayores de 43 años alcanzando más de la mitad, es decir un $12 \%$ del $21 \%$ total, y era menos frecuente en los adultos tempranos $(1 \%)$ e inexistente en los docentes jóvenes menores de 26 años $(0 \%)$.

\subsection{INTEGRACIÓN Y USOS DOCENTES DE LAS TIC}

Los usos tecnológicos encontrados se pueden agrupar en dos ámbitos: el personal y el académico.

\subsection{1 Ámbito personal:}

El $78 \%$ de los docentes usan con frecuencia las TIC en sus actividades personales para realizar proyectos, asesorías y consulta de información $(21 \%)$, leer noticias $(2 \%)$, realizar actividades de ocio $(5 \%)$ y cultivar relaciones sociales $(46 \%)$ con sus grupos primarios (familias, amigos, colegas) por medio del uso de computadores en la escuela y en sus hogares.

De este grupo de docentes que usan las TIC en el ámbito personal, vale la pena señalar que el $36 \%$ aún no ha integrado estos recursos a sus labores pedagógicas. Las razones para no integrarlas son variadas: algunos no tienen un buen dominio del computador, otros no la consideraron importante en los procesos educativos que adelantan desde sus planes de curso, y un remanente no sabe cómo articularlas a las dinámicas de aprendizaje con sus estudiantes.

\subsection{2 Ámbito académico:}

Se pudo determinar que el $42 \%$ de los docentes usan las TIC con fines académicos desde dos sub-ámbitos de aplicación: el área administrativa y el área pedagógica.

\subsubsection{Usos administrativos}

Los usos administrativos tienen que ver con los procesos de preparación, organización e implementación del plan de curso para lograr los propósitos establecidos. Dentro de la utilidad administrativa que los docentes otorgan a las TIC se destacan el uso de las TIC para: diseñar la programación de sus cursos y asignaturas (39\%); sistematizar la lista de asistencia y las calificaciones que van obteniendo sus estudiantes $(36 \%)$; actualizar la bibliografía y los materiales que aplican en sus cursos (34\%); preparar las clases $(33 \%)$ apoyados con herramientas ofimáticas; enviar información a sus estudiantes sobre el contenido de la asignatura $(31.7 \%)$; producción de guías de aprendizaje que utilizarán en el aula $(27 \%)$.

\subsubsection{Usos pedagógicos}

El uso pedagógico en estas escuelas tiene que ver con las diversas formas, estrategias y metodologías en que los docentes integran estos recursos tecnológicos en los procesos de aprendizaje con sus estudiantes. En términos generales se detectaron varios usos que fueron agrupados en cinco estrategias:

Como estrategia de socialización audiovisual:

Aquí las TIC son usadas para presentar videos y contenidos de la clase acompañado de textos, imágenes y diapositivas (41\%), presentar videos o películas $(41 \%)$, mostrar páginas de web pertinentes a la clase $(37 \%)$, permitir que los estudiantes expongan sus trabajos con ayudas audiovisuales $(31 \%)$ o socialicen sus tareas usando Blogs en la web (14\%).

Como soporte informacional y bibliográfico:

En este aspecto las TIC son utilizadas por los docentes para promover en sus clases el auto aprendizaje y la gestión informacional, específicamente las usan para el envío de documentos digitales de apoyo a las asignaturas $(26 \%)$, subir materiales que los estudiantes deben leer 
previa o posteriormente $(28 \%)$, buscar información científica en revistas, bases de datos y repositorios para fortalecer los contenidos de la clase $(26 \%)$ capacitar a sus estudiantes en la búsqueda de información académica pertinente al curso $(26 \%)$.

Como estrategia de interacción virtual complementaria:

En este aspecto los docentes buscan interaccionar con sus estudiantes fuera de las sesiones presenciales, esto con el fin de fortalecer el trabajo independiente y realizar actividades complementarias que permita a los estudiantes profundizar en los temas abordados. Las labores que más realizan con el uso de las TIC son: realización de diálogos en línea sobre temas de la asignatura $(25 \%)$ y realización de foros virtuales para plantear inquietudes o debatir sobre el asunto en cuestión (22\%).

Como estrategia de programación y simulación:

El docente genera nuevos ambientes virtuales de aprendizaje por medio de ejercicios prácticos que buscan que los estudiantes potencien el pensamiento lógico-creativo usando lenguajes de programación en la resolución de algoritmos $(25 \%)$, y el uso de programas de simulación $(18 \%)$ con el propósito de realizar experimentos y aplicaciones básicas en un entorno que simula el mundo real.

Como estrategia de evaluación formativa mediada:

Las TIC son usadas por algunos docentes para fortalecer el proceso de evaluación con sus estudiantes, mediante acciones concretas como: presentación de resultados de exámenes y parciales $(8 \%)$, realización de exámenes en línea (13\%) y retroalimentación virtual de los exámenes para aclarar dudas o solucionar inconformidades $(7 \%)$.

\subsection{TIPOLOGÍAS DE DOCENCIA}

Se encontraron diversas tipologías de docencia a partir de la forma en que los profesores de estas escuelas integran las TIC en sus procesos personales y académicos. Las tipologías fueron analizadas desde los postulados de Sánchez, Orjuela, Hooper y Rieber, emergiendo así:

El profesor tipo I (personalista): caracterizado por estar en un nivel de familiarización, ya que aunque poseen conocimientos sobre las TIC, la tendencia es a usarlas para sus labores personales $(36 \%)$. Todavía requieren formarse en competencias tecno-pedagógicas o desarrollar habilidades y experiencias que le permitan integrar estas tecnologías en sus labores pedagógicas.

El profesor tipo II (sistematizador): está en un nivel de utilización, en donde ha logrado integrar las TIC en labores académicas de carácter administrativo, en especial en el diseño de la programación de los cursos o asignaturas (39\%), la sistematización de las listas de asistencia y las notas de sus estudiantes (36\%).

El profesor tipo III (documentador): está en un nivel de preintegración en donde tiende a usar las TIC como soporte informacional y bibliográfico en la preparación de las clases (33\%), la producción de guías pertinentes a la clase $(27 \%)$, la búsqueda de información para la actualización de bibliografía y materiales que se usarán en la clase (34\%).

El profesor tipo IV (expositor): caracterizado por estar en un nivel de integración básica, en donde las TIC tiende a usarse como estrategia de socialización audiovisual en actividades de presentación de contenidos de la clase (31.1\%), presentación de películas y videos asociados al tema en cuestión $(29.7 \%)$ y para socializar resultados de exámenes o parciales $(6 \%)$.

El profesor tipo V (participativo): caracterizado por estar en un nivel de integración media, en donde la tendencia es a usar las TIC como estrategia de interacción virtual complementaria y de evaluación formativa mediada para fortalecer el trabajo independiente y la participación de los estudiantes en el proceso pedagógico. Las actividades más importantes que emergen de este tipo de docencia son: la realización de foros o debates en línea sobre temas del curso (26\%), la retroalimentación virtual de los resultados de exámenes y parciales (7.4\%), envío di- 
gital de lecturas previas para que participen en la clase $(21.1 \%)$, montaje de talleres o trabajos en la web $(22 \%)$ y permitir a los estudiantes exponer sus trabajos en la clase $(22 \%)$.

El profesor tipo VI (pragmatista): caracterizado por estar un nivel de integración avanzada, en donde la tendencia es a usar las TIC como estrategia de programación y simulación para potenciar el pensamiento lógico-matemático. Para tal fin, capacitan a sus estudiantes para que accedan a información de carácter científico en revistas especializadas y repositorios $(17.1 \%)$; capacitan a sus estudiantes para que aprendan a buscar y evaluar información pertinente a sus proyectos $(12.3 \%)$; ejercitan a sus estudiantes para que apliquen los conocimientos usando lenguajes de programación informáticos (25\%) y software simulación (18.2\%) para la resolución de problemas, lo que les permite estar inmersos en sus propios aprendizajes desde la experiencia práctica.

\subsection{LOGROS PERCIBIDOS EN LA INTE- GRACIÓN PEDAGÓGICA DE LAS TIC}

El $41 \%$ de los docentes percibieron mejoras en los procesos de integración pedagógica y en los objetivos educativos con la inserción de las TIC. Los logros concretos que identificaron en sus actividades académicas son:

El 8\% considera que gracias al uso de estas tecnologías ha aumentado el trabajo colaborativo e inter grupal dentro de las actividades de aula.

El 12\% afirma que el logro mayor con el uso de estos recursos es haber potenciado la participación y la interacción entre sus estudiantes.

El $21 \%$ de los docentes aseguran que sus estudiantes han potencializado mejor las competencias de gestión informacional reconociendo la necesidad de información en sus procesos de formación; de este grupo al menos el 11\% asegura que sus estudiantes saben buscar e identificar información pertinente para sus actividades de aprendizaje; el 10\% manifiesta que sus estudiantes saben identificar información relevante para resolver los problemas académicos derivados de la clase.

\subsection{DIFICULTADES EN LA INTEGRA- CIÓN PEDAGÓGICA DE LAS TIC}

Un 54\% restante de los docentes manifiestan no percibir ningún cambio o logro pedagógico a partir del uso de las TIC, por varias razones:

Algunos docentes no están integrando pedagógicamente las TIC: tal como se mencionó anteriormente, existen docentes que no tienen conocimiento en el uso de las TIC (22\%), otros no la están aplicando en sus actividades pedagógicas $(36 \%)$, y por eso es entendible que no perciban ningún logro pedagógico dentro de las escuelas.

La pérdida de la atención del estudiante en los fines centrales de la clase: los docentes reportan casos de estudiantes que se distraen con facilidad en el entorno digital durante las sesiones académicas (35\%), accediendo a redes sociales o a información ajena a los objetivos de la clase.

Viejas metodologías con nuevas tecnologías: Algunos docentes usan las TIC en sus actividades pedagógicas de presentación de diapositivas sobre los temas de la clase (29\%) manteniendo el modelo pedagógico tradicional centrado en la transmisión de información y de saberes.

La infraestructura tecnológica es ineficiente: algunos docentes consideraron que el servicio de internet es deficiente $(22 \%)$, manifiestan que a veces no es fácil acceder a los computadores de la escuela ya que la prioridad son las clases de tecnología informática (14\%), se quejan porque no se dispone de video beam o conexión de salida visual al alcance de ellos (6\%), la tecnología existente no abastece la demanda (12\%) y los computadores están obsoletos o desactualiza$\operatorname{dos}(7 \%)$.

\section{DISCUSIÓN}

El estudio realizado muestra que estas instituciones educativas desde la llegada del programa CPE han incrementado los usos de las TIC en la población docente, no solo porque ya disponen de estos recursos, sino también por los diversos espacios de capacitación que se han organizado. Esto ha permitido reducir el analfabetismo tecnológico a menos de la cuarta parte de esta po- 
blación y a que la gran mayoría de los docentes de estas escuelas perciban las TIC como aliadas del proceso pedagógico.

Este logro en materia de cobertura tecnológica y desarrollo profesoral, sigue siendo uno de los aspectos más significativos que han señalado los centros educativos en los diversos estudios que se han hecho en América Latina, y que han sido documentados ampliamente en los hallazgos de Artopoulos y Kozak (2011), Barrera y Linden (2009) y Salinas (2004); sin embargo, haber avanzado en cobertura y en el manejo técnico de estos recursos no significa que se han superado del todo los problemas de integración tecnológica, pues del 78\% de los docentes capacitados en el uso de las TIC (pese a que todos usan frecuentemente estas herramientas en sus labores personales), casi la mitad no ha extendido el uso de las TIC a las labores pedagógicas, que es donde la UNESCO $(2004 ; 2007)$ consideran que debe enfocarse la integración y la formación docente, lo cual significa que tener conocimiento o dominio tecnológico no garantiza su uso pedagógico.

En el caso de los docentes que si están usando pedagógicamente las TIC, hay que resaltar que la gran mayoría pertenecen a las distintas áreas del conocimiento que se imparten en estos centros educativos, lo cual para expertos como Batista (2007) es un gran avance en la tarea de transversalizar el uso de las TIC buscando desarrollar mejor los procesos de gestión de conocimiento y evitando que los usos tecnológicos no queden sectorizados a unas áreas especificas.

Precisamente los esfuerzos no se han dado de la forma esperada por la inexistencia de políticas de direccionamiento estratégico tendientes a lograr que el uso de las TIC sea una cultura democrática permanente, y porque aún persiste el monopolio de los laboratorios de informática, que centraliza el acceso tecnológico dando prioridad a los docentes de los cursos o asignaturas de tecnología informática.

En el mismo orden de ideas, se encontraron diferentes estrategias de uso a la par de diversas tipologías o modos de hacer docencia integrando las TIC en los procesos educativos. Estos hallazgos permiten afirmar que en estas escuelas coexisten múltiples culturas de integración tecno pedagógicas, algunas incluso guardan relación con los enfoques de integración descritos por la UNESCO (2008).

De esta manera es evidente que la gran mayoría de los docentes de estos centros educativos están en niveles de familiarización, pre integración o de integración básica, lo que hace que las tipologías de docencia tipo I, II, III y IV sean las más frecuentes, y el uso de las TIC tienda desde lo académico más hacia actividades administrativas de la clase, labores de gestión informacional, y como estrategia de socialización de información para facilitar los cursos o para brindar material digital a los estudiantes. Esta es una de las razones por las cuales la mayoría de los docentes, a pesar de que usan las TIC en la escuela, manifiesten no percibir ningún cambio en el modelo pedagógico ya que estas actividades están presentes también en la enseñanza tradicional y se hacían en antaño con otros tipos de tecnologías.

No obstante, es importante señalar que han emergido pedagógicamente dinámicas de docencia tipo V y VI con niveles de integración media y avanzada en donde se observan usos tecnológicos que modifican el modelo educativo tradicional, develando un interés en hacer de la clase y de la relación con el estudiante algo que trasciende el espacio físico del aula, lo unidireccional del aprendizaje y lo condicionado o limitado del tiempo formal en la escuela. En este tipo de docencia las TIC y en especial las basadas en internet, son usadas para fortalecer en los estudiantes la participación, la interlocución, el trabajo independiente, el aprendizaje interactivo y colaborativo, el análisis, la aplicación práctica del conocimiento y la capacidad para resolver de problemas.

\section{CONCLUSIONES}

A manera de síntesis es importante resaltar, que estas instituciones educativas desde la llegada de estos recursos tecnológicos entregados por el programa "Computadores para Educar" han mejorado la cobertura tecnológica y potencializado el uso de estos recursos a nivel personal 
y académico, gracias a que han sido acompañados de programas de formación profesoral.

Aun así para lograr un mayor aprovechamiento de las capacitaciones es pertinente que aprendan, no solo el manejo técnico, sino lo más importante, el desarrollo de habilidades docentes para una integración pedagógica innovadora de las TIC en donde prevalezcan dentro de las actividades de aula y extra aula, los significados y la capacidad de análisis por encima de la memorización de contenidos (Álvarez, Pérez y Suarez, 2008; Tenti, 2005); la gestión y evaluación de la información más que el dato en sí mismo (Roig, 2002), el proceso y no solo los resultados esperados; la interacción y el aprendizaje colaborativo más que el individualismo desin- tegrador (García, Basilotta y López, 2014); la duda más que la certeza, el contexto más que el pretexto, el dialogo más que el monologo, y el saber hacer en íntima relación con el saber ser, saber aprender y el saber convivir (Freire,1976; 1999; 2005).

Para lograr superar el analfabetismo tecnológico que aún persiste en estas instituciones educativa se deben crear: políticas para usar las TIC de forma educativa y democrática, ampliar el número de equipos de cómputo para cubrir la demanda escolar; mejorar la cobertura un acceso escolar abierto que haga posible que la conectividad no dependa exclusivamente de los computadores que están en las salas de cómputo.

\section{REFERENCIAS}

Álvarez, S., Pérez, A. y Suárez, M.(2008). Hacia un enfoque de la educación en competencias: Materiales de apoyo a la acción educativa (Serie). Principado de Asturias: Consejería de Educación y Ciencia, Servicio de Evaluación, Calidad y Ordenación Académica. Recuperado de: http://redined.mecd.gob.es/xmlui/bitstream/handle/11162/2576/01720082000075. pdf? sequence $=1$

Artopoulos, A. y Kozak, D. (2011). Topografías de la Integración de TICs en Latinoamérica. En D. Goldin, M. Kriscautzky, F. Perelman (Eds.). Las TIC en la escuela, nuevas herramientas para viejos y nuevos problemas (393-452). España: Editorial Océano.

Barrera, F. \& Linden, L. (2009). The use and misuse of computers in education: Evidence from a randomized Experiment in Colombia. Policy Working Research Paper, Series 4836. Recuperado de: http://elibrary.worldbank.org/doi/ pdf/10.1596/1813-9450-4836

Batista, M. (2007). Tecnologías de la información y la comunicación en la escuela: trazos, claves y oportunidades para su integración pedagógica. Buenos Aires: Ministerio de Educación, Ciencia y Tecnología de la Nación.

Computadores para Educar. (2012). Informe de gestión. Recuperado de: http://www.computadoresparaeducar.gov.co/inicio/sites/default/files/documentos/Informe\%20de\%20gestion\%202012\%20.pdf

Freire, P. (1976). La educación como práctica de la libertad. Madrid: Siglo XXI de España editores.

Freire, P. (1999). La importancia de leer y el proceso de liberación. Buenos Aires: Siglo XXI, editores argentina.

Freire, P. (2005). Pedagogía del oprimido. Buenos Aires: Siglo XXI editores.

García, A., Basilotta, V. y López, C. (2014). Las TIC en el aprendizaje colaborativo en el aula de Primaria y Secundaria. Comunicar, 21 (42), 65-74.

Hooper, S. \& Rieber, L. (1995). Teaching with technology. In A.C Ornstein (Ed.). Teaching: Theory into (154-170). Needham Heights, MA: Allyn and Bacon. 
Jaramillo, P., Castañeda, P., \& Pimienta, M. (2009). Qué hacer con la tecnología en el aula: inventario de usos de las TIC para aprender y enseñar. Educación y educadores, 12 (2), 159-179.

Moreno, J., Anaya, S., Benavides, P. y Hernández, U. (2011). Los proyectos pedagógicos de Aula para la integración de las TIC. Popayan: Universidad del Cauca.

Mejía, M. y Bernal, P. (2003). Computadores para Educar: Enriqueciendo la Formación de las Nuevas Generaciones de Colombianos. Ottawa: Instituto para la Conectividad en las Américas. Recuperado de: http://web.idrc.ca/uploads/userS/11776948801Computadores-Documento_Integrado.pdf

Orjuela, D. (2010). Acercamiento a la integración curricular de las TIC. Praxis y saber, 1(2), 111-136.

Pardo, M. (2005). Experiencias significativas para poblaciones vulnerables. Bogotá: Ministerio de Educación Nacional. Recuperado de: http://www.colombiaaprende.edu.co/html/mediateca/1607/articles-88730_archivo.pdf

PNUD (2011). Cartagena de Indias: retos y propuestas para la construcción colectiva de un programa de gobierno. Bogotá: Programa de las Naciones Unidas para el Desarrollo.

Rodríguez, C., Sánchez, F. y Márquez, J. (2011). Impacto del Programa Computadores para Educar en la deserción estudiantil, el logro escolar y el ingreso a la educación superior. Serie Documentos Cede. Bogotá: Universidad de los Andes. Recuperado de: http://economia.uniandes.edu.co/content/download/37488/319392/file/dcede2011-15.pdf

Roig, R. (2002). Las Nuevas Tecnologías aplicadas a la educación: elementos para una articulación didáctica de las Tecnologías de la Información y la Comunicación. Alcoy: Marfil.

Salinas, J. (2004). Innovación docente y uso de las TIC en la enseñanza universitaria. Universidad y sociedad del conocimiento, 1 (1), 1-16.

Sánchez, J. (2003). Integración curricular de TICs: conceptos y modelos. Enfoques educacionales, 5 (1), 51-65.

Tenti, F. (2005). La condición docente. Análisis comparado de la Argentina, Brasil, Perú y Uruguay. Buenos Aires: Siglo Veintiuno Argentina Editores, Fundación OSDE.

Trahtemberg, L. (2001). El impacto previsible de las nuevas tecnologías en la enseñanza y la organización escolar. En H. Gianini y J. Allende (Eds.). Análisis de prospectivas de la educación en América Latina y el Caribe: Seminario sobre prospectivas de la educación en la región de América Latina y el Caribe (292-320). Santiago de Chile: UNESCO.

UNESCO. (2004). Las tecnologías de la información y la comunicación en la formación docente. París: Organización de las Naciones Unidas para la Educación, la Ciencia y la Cultura. Recuperado de: http://unesdoc.unesco.org/ images/0012/001295/129533s.pdf

UNESCO (2007). Educación de Calidad para Todos: un asunto de derechos humanos. Santiago de Chile: OREALC/ UNESCO. Recuperado de: http://unesdoc.unesco.org/images/0015/001502/150272s.pdf

UNESCO. (2008). Estándares de competencia en TIC para docentes. Londres: Organización de las Naciones Unidas para la Educación, la Ciencia y la Cultura. Recuperado de: http://www.oei.es/tic/UNESCOEstandaresDocentes.pdf 